\title{
NEW UPPER BOUNDS ON THE ORDER OF CAGES ${ }^{1}$
}

\author{
F. LAZEBNIK \\ Department of Mathematical Sciences \\ University of Delaware, Newark, DE 19716, USA; fellaz@math.udel.edu \\ V. A. USTIMENKO \\ Department of Mathematics and Mechanics \\ University of Kiev, Kiev 252127, Ukraine; vau@trion.kiev.ua \\ A. J. WOLDAR \\ Department of Mathematical Sciences \\ Villanova University, Villanova,PA 19085,USA; woldar@ucis.vill.edu \\ Submitted: August 1, 1996; Accepted: November 21, 1996. \\ Dedicated to Herbert $S$. Wilf on the occasion of his $65^{\text {th }}$ birthday
}

\begin{abstract}
Let $k \geq 2$ and $g \geq 3$ be integers. A $(k, g)$-graph is a $k$-regular graph with girth (length of a smallest cycle) exactly $g$. A $(k, g)$-cage is a $(k, g)$-graph of minimum order. Let $v(k, g)$ be the order of a $(k, g)$-cage. The problem of determining $v(k, g)$ is unsolved for most pairs $(k, g)$ and is extremely hard in the general case. It is easy to establish the following lower bounds for $v(k, g): v(k, g) \geq \frac{k(k-1)(g-1) / 2-2}{k-2}$ for $g$ odd, and $v(k, g) \geq \frac{2(k-1)^{g / 2}-2}{k-2}$ for $g$ even. The best known upper bounds are roughly the squares of the lower bounds. In this paper we establish general upper bounds on $v(k, g)$ which are roughly the $3 / 2$ power of the lower bounds, and we provide explicit constructions for such $(k, g)$-graphs.
\end{abstract}

Mathematical Reviews Subject Numbers: 05C35, 05C38. Secondary: 05D99.

\section{Introduction}

All graphs in this paper are assumed to be simple (undirected, no loops, no multiple edges). Let $k \geq 2$ and $g \geq 3$ be integers. A $(k, g)$-graph is a $k$-regular

1 This research was supported by NSF grants DMS-9115473 and DMS-9622091. A.J. Woldar was additionally supported by a Villanova University Faculty Research Grant. V.A. Ustimenko was additionally supported in part by INTAS-93-2530 and INTAS-94-3420. F. Lazebnik was additionally supported by a University of Delaware Research Award. 
graph with girth (length of a smallest cycle) exactly $g$. A $(k, g)$-cage is a $(k, g)$-graph of minimum order. The problem of determining the order $v(k, g)$ of a $(k, g)$-cage is unsolved for most pairs $(k, g)$ and is extremely hard in the general case. By counting the number of vertices in the breadth-first-search tree of a $(k, g)$-graph, one easily establishes the following lower bounds for $v(k, g)$ :

$$
v(k, g) \geq \begin{cases}\frac{k(k-1)^{(g-1) / 2}-2}{k-2} & \text { for } g \text { odd } \\ \frac{2(k-1)^{g / 2}-2}{k-2} & \text { for } g \text { even }\end{cases}
$$

Graphs whose orders achieve these lower bounds are very special and possess many remarkable properties. Though there is no complete agreement on terminology, they are often referred to as "Moore graphs" when $g$ is odd, and "regular generalized polygons" when $g$ is even. For references on cages, see Wong [23] Section 6.9 of Brouwer, Cohen and Neumaier [6], Chapter 23 of Biggs [2], and Chapter 6 of Holton and Sheehan [8], For a survey of results on cubic cages $(k=3)$ with girth at most 20, see Royle [18].

Finding upper bounds for $v(k, g)$ is a far more difficult affair; indeed, even the fact that $v(k, g)$ is finite is nontrivial to prove. This was settled by Sachs [19] who showed by explicit construction that $(k, g)$-graphs of finite order exist. In the same year, Erdős and Sachs [7] gave, without explicit construction, a much smaller general upper bound on $v(k, g)$. (As was pointed by Alon in [1, p. 1752], although their proof does supply a polynomial time algorithm for constructing graphs which provide the upper bound, their graphs are not really explicit in the sense that it is not clear how to decide efficiently whether or not two prescribed vertices of such a graph are adjacent.) Their result was later improved, though slightly, by Walther $[21],[22]$ and by Sauer [20]. The following upper bounds are due to Sauer [20]:

$$
v(k, g) \leq \begin{cases}2(k-1)^{g-2} & \text { for } g \text { odd and } k \geq 4, \text { and } \\ 4(k-1)^{g-3} & \text { for } g \text { even and } k \geq 4\end{cases}
$$

Note that these upper bounds are roughly the squares of the previously indicated lower bounds.

In this paper we establish general upper bounds on $v(k, g)$ which are roughly the $3 / 2$ power of the lower bounds, and we provide explicit constructions for such $(k, g)$-graphs. The main results are described below. 
Theorem A. Let $k \geq 2$ and $g \geq 5$ be integers, and let $q$ denote the smallest odd prime power for which $k \leq q$. Then

$$
v(k, g) \leq 2 k q^{\frac{3}{4} g-a},
$$

where $a=4,11 / 4,7 / 2,13 / 4$ for $g \equiv 0,1,2,3 \bmod 4$, respectively. 5 .

The upper bounds in (2) are better the ones provided in (1) for all $k \geq 5$ and $g \geq$ For $k \geq t \geq 3$ and $q \equiv 1 \bmod t,(k, 2 t)$-graphs of orders at least as large as the upper bound in (2) were constructed in[9] by Füredi, Seress, and the authors. The fact that the orders of these constructions actually meet the upper bound in (2) for $q$ odd follows from [13]. The constructions we introduce in this paper are independent of the relative magnitudes of $k$ and $g$.

Constructions. For all $k \geq 3$ and $g \geq 6$, g even, we explicitly construct a $(k, g)$ graph of order

$$
g\left(1+(k-2) k q^{g-5-\left\lfloor\frac{g-3}{4}\right\rfloor}\right)
$$

for all $k \geq 3$ and $g \geq 5, g$ odd, we explicitly construct a $(k, g)$-graph of order

$$
(g+1)\left(1+(k-2) k q^{g-4-\left\lfloor\frac{g-2}{4}\right\rfloor}\right) .
$$

In either case, $q$ is the smallest odd prime power for which $k \leq q$.

Though the upper bounds on $v(k, q)$ provided by these constructions are not as good as the ones given in Theorem A, they are better than the bounds in (1) for all $k \geq 7$ and $g \geq 11$ when $g$ is odd, and $g \geq 8$ when $g$ is even. With some additional restrictions on $k$ and $g$ these constructive upper bounds can be improved still further.

By Chebyshev's Theorem, for a fixed integer $k \geq 3$ there is always a prime between $k$ and $2 k-2$. For any $\epsilon>0$ and $k \geq k_{0}(\epsilon)$, this interval can be narrowed to $\left[k, k+k^{\left.\frac{3}{5}+\epsilon\right]}\right.$, see $[17$, p. 131]. Thus the upper bounds from (2), (3), and (4) are roughly the $3 / 2$ power of the indicated lower bounds. 


\section{Preliminary Results and Proof of Theorem A}

The possibility of improving the upper bounds in (1) for $(k, g) \in A \times B$, where both $A$ and $B$ are certain infinite subsets of positive integers, became apparent after the discoveries of certain special infinite families of graphs which we describe below (see F1, F2 and F3).

For a fixed integer $k \geq 3$, let $\left\{G_{i}\right\}_{i \geq 1}$ be a family of $k$-regular graphs of increasing order $v_{i}$. Let $g_{i}$ denote the girth of $G_{i}$. The family $\left\{G_{i}\right\}$ is called a family of graphs with large girth if

$$
g_{i} \geq \gamma \log _{k-1}\left(v_{i}\right)
$$

for some positive constant $\gamma$ and all $i \geq 1$. The lower bound for $v(k, g)$ shows that $\gamma \leq 2$, but no infinite family has been found for which $\gamma=2$.

F1. Margulis [16] and, independently, Lubotzky, Phillips and Sarnak [15] came up with similar examples of graphs with $\gamma \geq 4 / 3$ and arbitrary large valency (they turned out to be so-called Ramanujan graphs). These are Cayley graphs of the group $P G L_{2}\left(Z_{q}\right)$ with respect to a set of $p+1$ generators, where $p$ and $q$ are distinct primes, each congruent to $1 \bmod 4$, with the Legendre symbol $\left(\frac{p}{q}\right)=-1$. Denoted by $X^{p, q}$, they are $(p+1)$-regular bipartite graphs of order $q\left(q^{2}-1\right)$. Margulis [16] and, independently, Biggs and Boshier 3] showed that the asymptotic value of $\gamma$ for the graphs $X^{p, q}$ is exactly $4 / 3$. Moreover, in both papers an explicit formula for the girth $g\left(X^{p, q}\right)$ of $X^{p, q}$ was found. To state their results (formulae (5), below) we first need the following definition.

Call an integer good if it is not of the form $4^{\alpha}(8 \beta+7)$ for any nonnegative integers $\alpha, \beta$. By a theorem of Legendre, good numbers are precisely those which are representable as sums of three squares. Then

$$
g\left(X^{p, q}\right)= \begin{cases}2\left\lceil 2 \log _{p} q\right\rceil & \text { if } p^{\left\lceil 2 \log _{p} q\right\rceil}-q^{2} \text { is good } \\ 2\left\lceil 2 \log _{p} q+\log _{p} 2\right\rceil & \text { otherwise. }\end{cases}
$$

F2. In 10], Lazebnik and Ustimenko constructed the family of graphs $D(n, q)$, $n \geq 2, q$ a prime power, for which $\gamma \geq \log _{q}(q-1)$. Graphs $D(n, q)$ are $q$-regular of order $2 q^{n}$ and girth at least $n+4$ (respectively, $n+5$ ) for $n$ even (respectively, $n$ odd). These are defined as follows.

Let $q$ be a prime power, and let $P$ and $L$ be two copies of the countably infinite dimensional vector space over $G F(q)$. In order to distinguish between vectors from 
$P$ and $L$ we use parentheses and brackets: $x \in P$ will be written as $(x)$, and $y \in L$ as $[y]$. Adopting the notation for coordinates of points and lines introduced in [10], namely,

$$
\begin{gathered}
(p)=\left(p_{1}, p_{11}, p_{12}, p_{21}, p_{22}, p_{22}^{\prime}, p_{23}, \ldots, p_{i i}, p_{i i}^{\prime}, p_{i, i+1}, p_{i+1, i}, \ldots\right), \\
{[l]=\left[l_{1}, l_{11}, l_{12}, l_{21}, l_{22}, l_{22}^{\prime}, l_{23}, \ldots, l_{i i}, l_{i i}^{\prime}, l_{i, i+1}, l_{i+1, i}, \ldots\right],}
\end{gathered}
$$

we define an infinite bipartite graph $D(q)$ with the vertex set $P \cup L$ as follows. We say $(p)$ is adjacent to $[l]$ if the following relations on their coordinates hold:

$$
\begin{aligned}
& l_{11}-p_{11}=l_{1} p_{1} \\
& l_{12}-p_{12}=l_{11} p_{1} \\
& l_{21}-p_{21}=l_{1} p_{11} \\
& l_{i i}-p_{i i}=l_{1} p_{i-1, i} \\
& l_{i i}^{\prime}-p_{i i}^{\prime}=l_{i, i-1} p_{1} \\
& l_{i, i+1}-p_{i, i+1}=l_{i i} p_{1} \\
& l_{i+1, i}-p_{i+1, i}=l_{1} p_{i i}^{\prime}
\end{aligned}
$$

(The last four relations are defined for all $i \geq 2$.) For each positive integer $n \geq 2$ we obtain a finite bipartite graph $D(n, q)$ as follows. First, $P_{n}$ and $L_{n}$ are obtained from $P$ and $L$, respectively, by simply projecting each vector onto its $n$ initial coordinates. Let the set of vertices of $D(n, q)$ be $P_{n} \cup L_{n}$. Adjacency in $D(n, q)$ is now defined in terms of the first $n-1$ relations of (6), and no others. (Note that these relations involve only the first $n$ coordinates of vectors from $P \cup L$, so apply unambiguously to vectors from $P_{n} \cup L_{n}$.)

In [12], Lazebnik, Ustimenko and Woldar showed that the graphs $D(n, q)$ are disconnected for $n \geq 6$ and that their connected components $C D(n, q)$ (all isomorphic) have order at most $2 q^{n-\left\lfloor\frac{n+2}{4}\right\rfloor+1}$. As $C D(n, q)$ and $D(n, q)$ have the same girth, it follows that the graphs $C D(n, q)$ form a family for which $\gamma \geq \frac{4}{3} \log _{q}(q-1)$. With few exceptions, these graphs provide the best known asymptotic lower bound for the greatest number of edges in graphs of their order and girth. Later, in [13], the authors proved that for $q$ odd, the order of $C D(n, q)$ is exactly $2 q^{n-\left\lfloor\frac{n+2}{4}\right\rfloor+1}$. Combining this with a result from [9] on the existence of a girth cycle of length $n+5$ in $D(n, q)$ for all odd $n$ and infinitely many $q$, we get that the corresponding subfamily of graphs $C D(n, q)$ satisfies $\gamma=\frac{4}{3} \log _{q}(q-1)$.

An important property of graphs $D(n, q)$ and $C D(n, q)$ is that they contain a special type of induced subgraph. These were introduced by the authors in [11] and 
can be described as follows. Let $R, S \subseteq G F(q)$, where $|R|=r \geq 1$ and $|S|=s \geq 1$, and let

$$
\begin{aligned}
& P_{R}=\left\{(p) \in P_{n} \mid p_{1} \in R\right\} \\
& L_{S}=\left\{[l] \in L_{n} \mid l_{1} \in S\right\} .
\end{aligned}
$$

We define $D(n, q, R, S)$ to be the subgraph of $D(n, q)$ induced on $P_{R} \cup L_{S}$. For fixed $(p) \in P_{R}$ and arbitrary $x \in S$, there exists a unique neighbor $[l]$ of $(p)$ in $D(n, q, R, S)$ with first coordinate $l_{1}$ equal to $x$. Since there are precisely $s$ choices for $x$, the adjacency relations (6) imply that the degree of $(p)$ in $D(n, q, R, S)$ is $s$. Similarly, for any $[l] \in L_{S}$ the degree of $[l]$ in $D(n, q, R, S)$ is $r$. Therefore $D(n, q, R, S)$ is an induced subgraph of $D(n, q)$ with bi-degree $s, r$ (every vertex from $P_{R}$ has degree $s$ and every vertex from $L_{S}$ has degree $r$ ). Analogously, we denote by $C D(n, q, R, S)$ the induced subgraph of $C D(n, q)$ obtained by performing this procedure on $C D(n, q)$. As with $D(n, q, R, S)$, the graph $C D(n, q, R, S)$ has bi-degree $s, r$.

F3. In $[9]$ Füredi and Seress, together with the authors, showed that for all $r, s, t \geq$ 2 , there exists a bipartite graph of girth $2 t$ and bi-degree $r, s$. They also showed that for all $r, s \geq t \geq 3$, there exists a bipartite graph with bi-degree $r, s$ and girth $2 t$ of order $(r+s) q^{2 t-6}$, where $q \geq r, s$ is the smallest prime power of the form $1+m t$ for some integer $m \geq 1$. This graph was constructed as $D(2 t-5, q, R, S)$ where $R$ and $S$ were chosen in a special way. As follows from[13], $C D(2 t-5, q, R, S)$ is an $(r, 2 t)$-graph of order $2 r q^{2 t-5-\left\lfloor\frac{2 t-3}{4}\right\rfloor}$ when $r=s \geq t \geq 3$.

Each of the three families described in F1-F3 can be used to improve the upper bound in (1) for $(k, g) \in A \times B$, where both $A$ and $B$ are infinite subsets of positive integers. The authors expended considerable energy attempting to extend these improvements to a set $A \times B$ where $A$ and $B$ are not just infinite subsets of positive integers but have finite complements, e.g. $A=\left\{a: a \geq a_{0}\right\}$ and $B=\left\{b: b \geq b_{0}\right\}$, for some positive integers $a_{0}, b_{0}$. We tried to do this constructively by considering special subgraphs of $X^{p, q}$ and $C D(n, q)$.

A natural way to find a $k$-regular subgraph in the Cayley graph $X_{p, q}$ is to restrict the set of $p+1$ generators to a symmetric $k$-element subset. The difficulty is to do this in such a way that the girth of the subgraph equals a prescribed even integer $g$. Formulae (5) are not of much help here. Another drawback to this approach is that the subgraph is not necessarily induced, which decreases its chances of having a small number of vertices in comparison to its valency and girth.

The situation is better with graphs $C D(n, q)$. Indeed, the subgraphs $C D(n, q, R, R),|R|=k \leq q$, are not only $k$-regular but induced. Though we seem to 
have some additional control on measuring the girth of these subgraphs, an exact determination is very difficult. Even in those cases where we know $g(C D(n, q))$ (equivalently, $g(D(n, q))$ ) explicitly, we have not yet found a way to either preserve this girth, or trace its change, when passing to induced subgraphs with given but arbitrary valency $k$. All that we know with certainty is the obvious fact that these subgraphs have girth at least $n+5$ for $n$ odd and $n+4$ for $n$ even, as the same property holds for the graphs $C D(n, q)$.

Our prospects of solving this problem looked rather grim until a few months ago when new results came in a rather unexpected way. First, we discovered a simple way to construct families of $(k, g)$-graphs from those of $k$-regular graphs of girth at least $g$. Applying this construction to the graphs $C D\left(2\left\lfloor\frac{g+1}{2}\right\rfloor-5, q\right)$, we obtained the upper bounds given in (3) and (4). Soon after, we realized that we had overlooked a beautiful result of Erdős and Sachs, which asserts that the orders of $k$ regular graphs of girth at least $g$ provide upper bounds on the orders of $(k, g)$-cages. Namely,

Theorem 2.1 [7] Let $G$ be a k-regular graph of girth at least $g$ having the least number of vertices. Then the girth of $G$ is $g$ and the diameter of $G$ is at most $g$.

A proof of this theorem can be found in [14, pp. 66, 384, 385], see also the references therein. Applying it to the $k$-regular subgraphs $C D(n, q, R, R)$ of $C D(n, q)$, where $n=2\left\lfloor\frac{g+1}{2}\right\rfloor-5$ and $|R|=k \leq q$, one immediately obtains the general nonconstructive upper bounds given in $(2)$ which are better than those given in $(1)$. The modulo 4 classification is trivial; thus Theorem A is proven.

\section{Constructions}

As we mentioned in the Introduction, for $k \geq t \geq 3$ and $q$ the smallest prime power for which $q \geq k$ and $q \equiv 1 \bmod t,(k, 2 t)$-graphs whose orders are at least as large as the upper bound in (2) were constructed by Füredi, Seress and the authors, see [9]. The fact that the orders of these constructions actually meet the upper bound in (2) for $q$ odd follows from [13]. Therefore the constructions we present below are interesting mainly for $k<g / 2$. Before proceeding, we mention that in every case the graphs we construct can be viewed, roughly, as being formed by appending an appropriate number of high girth graphs to a "central" $g$-cycle.

Case 1: $g$ even. Let $k \geq 3$ be an integer, $g=2 s \geq 4$ an even integer, and $C=v_{1} v_{2} \ldots v_{2 s} v_{1}$ a cycle of order $g$. Let $\left\{H_{i j}\right\}$ be an arbitrary family of $k$-regular 
graphs, each of order $v$ and girth at least $g, 1 \leq i \leq s, 1 \leq j \leq k-2$. In each $H_{i j}$ choose, arbitrarily, a "distinguished" edge and denote it by $a_{i j} b_{i j}$. Now, denote by $H_{i j}^{*}$ the graph obtained from $H_{i j}$ by deleting edge $a_{i j} b_{i j}$. Finally, form the graph $H$ by adjoining the graphs $H_{i j}^{*}$ to the cycle $C$ in the following manner: For each $1 \leq i \leq s, 1 \leq j \leq k-2$, adjoin vertex $a_{i j}$ (respectively, $b_{i j}$ ) of graph $H_{i j}^{*}$ to vertex $v_{2 i-1}$ (respectively, $v_{2 i}$ ) of $C$. It is trivial to see that $H$ is a $k$-regular graph of order $g+s(k-2) v$ and that the girth of $H$ is at most $g$.

Let us show that $g(H)=g$. Let $K$ be a cycle in $H$ of order less than $g$. Then $K$ must contain at least one pair of edges of the form $a_{i_{0} j_{0}} v_{2 i_{0}-1}, b_{i_{0} j_{0}} v_{2 i_{0}}$, as otherwise $K$ would be a cycle in either $C$ or $H_{i j}$ for some $i, j$. But now the portion of $K$ which lies in $H_{i_{0} j_{0}}^{*}$, together with the edge $a_{i_{0} j_{0}} b_{i_{0} j_{0}}$, forms a cycle in $H_{i_{0} j_{0}}$ of order less than $g$.

Now, taking each $H_{i j}$ to be the $k$-regular subgraph $C D(g-5, q, R, R)$ of $C D(g-$ $5, q$ ), where $q$ is the smallest odd prime power for which $q \geq k=|R|$, we obtain the order of $H$ as appears in $(3)$.

Case 2: $g$ odd. Let $k \geq 3$ be an integer, $g=2 s-1 \geq 3$ an odd integer, and $C=v_{1} v_{2} \ldots v_{2 s-1} v_{1}$ a cycle of order $g$. Adjoin a new vertex $v_{2 s}$ to $v_{2 s-1}$ but to no other vertex of $C$.

With $\left\{H_{i j}\right\}, a_{i j} b_{i j}$, and $H_{i j}^{*}(1 \leq i \leq s, 1 \leq j \leq k-2)$ defined exactly as in Case 1, we form $H$ as follows: For each $1 \leq i \leq s-1,1 \leq j \leq k-2$, adjoin vertex $a_{i j}$ (respectively, $b_{i j}$ ) of graph $H_{i j}^{*}$ to vertex $v_{2 i-1}$ (respectively, $v_{2 i}$ ) of $C$. Next for each $1 \leq j \leq k-3$, adjoin vertex $a_{s j}$ (respectively, $b_{s j}$ ) of graph $H_{s j}^{*}$ to vertex $v_{2 s-1}$ of $C$ (respectively, vertex $v_{2 s}$ ). Finally, adjoin both vertices $a_{s, k-2}$ and $b_{s, k-2}$ of $H_{s, k-2}^{*}$ to vertex $v_{2 s}$.

It is routine to check that $H$ is $k$-regular of order $g+1+s(k-2) v$, and that the girth of $H$ is at most $g$. One uses an argument similar to that given in Case 1 to establish that $g(H)=g$. Taking each $H_{i j}$ to be the $k$-regular subgraph $C D(g-4, q, R, R)$ of $C D(g-4, q)$, where $q$ is the smallest odd prime power for which $q \geq k=|R|$, we obtain the order of $H$ as appears in (4).

Remark. It is easy to see that the diameter of a $k$-regular graph of order $v$ is at least $\log _{k-1} v$ and that the random $k$-regular graph has diameter close to this lower bound, see [5, Ch. X3]. Though several explicit constructions of families of $k$ regular graphs with diameters close to $\log _{k-1} v$ are known $[5, \mathrm{Ch}$. X1] these all have small girth. The problem of constructing infinite families of graphs of large girth 
and small diameter (i.e. with diameter at most $c \log _{k-1} v, c \geq 1$ a constant) is far from trivial. For the Ramanujan graphs described in F1, diameter is known to be at most $2 \log _{k-1} v+2$, and the proof of this fact is not simple. The upper bound on the girth of graphs $C D(n, q)$, together with the statement in Theorem 2.1 regarding diameter of cages, implies that for every $k \geq 3$ there exists a family of graphs of large girth $\left\{G_{i}\right\}_{i \geq 1}$ such that $g\left(G_{i}\right) \geq \frac{4}{3} \log _{k-1} v_{i}$ and $\operatorname{diam}\left(G_{i}\right) \leq \frac{4}{3} \log _{k-1} v_{i}$. We conjecture that the diameter of graphs $C D(n, q)$ is within an additive constant of this bound. More precisely,

Conjecture. There exists a positive constant $C$ such that for all integers $n \geq 2$ and all prime powers $q$,

$$
\operatorname{diam}(C D(n, q)) \leq\left(\log _{q-1} q\right) n+C
$$




\section{References}

1. N. Alon, Tools from Higher Algebra, in: Handbook of Combinatorics, Volume II (edit. R. L. Graham, M. Grötschel, L. Lovász), MIT Press, North-Holland, New York, 1995.

2. N. L. Biggs, Algebraic Graph Theory (2nd ed.), Cambridge University Press, 1993.

3. N. L. Biggs and A. G. Boshier, Note on the girth of Ramanujan graphs, Journal of Combinatorial Theory Series B, vol. 49 , 190-194 (1990).

4. B. Bollobás, Extremal Graph Theory, Academic Press, London, 1978.

5. B. Bollobás, Random Graphs, Academic Press, London, 1985.

6. A. E. Brouwer, A. M. Cohen, A. Neumaier, Distance-Regular Graphs, SpringerVerlag, Heidelberg - New York, 1989.

7. P. Erdős and H. Sachs, Reguläre Graphen gegebener Taillenweite mit minimaler Knotenzahl, Wiss. Z. Univ. Halle Martin Luther Univ. Halle-Wittenberg Math.-Natur.Reine 12 (1963), 251-257.

8. D. A. Holton, J. Sheehan, The Petersen Graph, Australian Mathematical Society, Lecture Notes 7, Cambridge University Press, 1993.

9. Z. Füredi, F. Lazebnik, Á. Seress, V. A. Ustimenko, A. J. Woldar, Graphs of prescribed girth and bi-degree, Journal of Combinatorial Theory Ser. B 64 (2) (1995), 228-239.

10. F. Lazebnik, V. A. Ustimenko, Explicit construction of graphs with arbitrary large girth and of large size, Discrete Applied Mathematics 60 (1995), 275-284.

11. F. Lazebnik, V. A. Ustimenko, A. J. Woldar, New constructions of bipartite graphs on $m, n$ vertices, with many edges, and without small cycles, Journal of Combinatorial Theory Ser. B 61 (1) (1994), 111-117.

12. F. Lazebnik, V. A. Ustimenko, A. J. Woldar, A new series of dense graphs of high girth, Bulletin of the AMS 32 (1) (1995), 73-79.

13. F. Lazebnik, V. A. Ustimenko, A. J. Woldar, A characterization of the components of the graphs $D(k, q)$, Discrete Mathematics 157 (1996) 271-283.

14. L. Lovász, Combinatorial Problems and Exercises, North-Holland, Amsterdam, 1979. 
15. A. Lubotzky, R. Phillips, R. Sarnak, Ramanujan graphs, Combinatorica 8 (3) (1988), 261-277.

16. G. A. Margulis, Explicit group-theoretical construction of combinatorial schemes and their application to the design of expanders and concentrators, Journal of Problems of Information Transmission (1988), 39-46.

17. H. L. Montgomery, Topics in Multiplicative Number Theory, Lecture Notes in Mathematics 227, Springer Verlag, New York, 1971.

18. G. Royle, Cubic cages, http://www.ss.uwa.edu.au/gordon/cages/index.html.

19. H. Sachs, Regular graphs with given girth and restricted circuits, J. London Math. Society 38 (1963), 423-429.

20. N. Sauer, Extremaleigenschaften regulärer Graphen gegebener Taillenweite, I and II, Sitzungsberichte Österreich. Acad. Wiss. Math. Natur. Kl., S-B II, 176 (1967), 9-25; 176 (1967), 27-43.

21. H. Walther, Eigenschaften von regulären Graphen gegebener Taillenweite und minimaler Knotenzahl, Wiss. Z. Ilmenau 11 (1965), 167-168.

22. H. Walther, Über reguläre Graphen gegebener Taillenweite und minimaler Knotenzahl, Wiss. Z. Techn. Hochsch. Ilmenau 11 (1965), 93-96.

23. P.-K. Wong, Cages - A Survey, Journal of Graph Theory Vol. 6 (1982), 1-22. 